\title{
NEUROPSYCHOPHARMACOLOGY REVIEWS Sex differences in neuroimmunity as an inherent risk factor
}

\author{
Margaret M. McCarthy $\mathbb{D}^{1}$ \\ Identifying and understanding the sources of inherent risk to neurodevelopmental disorders is a fundamental goal of neuroscience. \\ Being male or being exposed to inflammation early in life are two known risk factors, but they are only infrequently associated with \\ each other. Cellular and molecular mechanisms mediating the masculinization of the brain in animal models reveal a consistent role \\ for inflammatory signaling molecules and immune cells in the healthy male brain. Why this is so remains in the realm of speculation \\ but may have its origins in the maternal immune system. Masculinization of the brain occurs during a restricted critical period that \\ begins in utero and overlaps with the sensitive period during which maternal immune activation negatively impacts the developing \\ brain. The convergence of maleness and early life inflammation as risk factors for neuropsychiatric disorders compels us to consider \\ whether sexual differentiation of the brain in males creates an inherent and greater risk than that experienced by females.
}

Neuropsychopharmacology (2019) 44:38-44; https://doi.org/10.1038/s41386-018-0138-1

\section{INTRODUCTION}

Neuroimmunology was traditionally considered a sub-discipline of neuroscience that focused on brain injury and autoimmune diseases. This has changed dramatically in recent years with the realization that the immune system of the brain is far more complex than originally appreciated and far more integral to almost every aspect of neural functioning, in both health and disease, than originally thought. Likewise for the sub-discipline of neuroendocrinology and the sub-sub-discipline of sex differences in the brain, which are now appreciated to be far more complex than originally appreciated and far more integral to almost every aspect of neural functioning, in both health and disease, than originally thought. Further similarities are found in the discovery that gestational exposure to inflammation is an important yet poorly understood contributor to the relative risk of a developmental neuropsychiatric disorder [1], and that being male is also a major but poorly understood risk factor for all developmental disorders [2]. Sex differences in the brain are programmed in utero during a critical period for sexual differentiation (Fig. 1). The parallel lines of in utero inflammation and in utero sexual differentiation are now crossing with the emerging evidence that the cellular and molecular mechanisms mediating masculinization of the brain are dominated by immune and inflammatory processes, thereby generating the thesis of this review, that an inherent difference in the neuroimmune system of developing males versus females may increase the risk for dysregulated brain development.

\section{SEX DETERMINATION VERSUS SEXUAL DIFFERENTIATION}

Sex determination is the process whereby a bipotential gonadal anlage differentiates into either an ovary or a testis in the early days of embryonic development. In mammals, the decision point is the expression of the Sry gene located on the $Y$ chromosome and which codes for Tdf, the testis determining factor. There is no homolog for Sry on the $X$ chromosome and the absence of this gene results in the formation of an ovary [3]. Sexual differentiation refers to all subsequent processes that are downstream of the gonads, including the establishment of a male versus female reproductive tract, secondary sexual characteristics and aspects of neuroanatomy and physiology. From a reproductive standpoint, the most important components of sexual differentiation of the brain are the programming of the neural control of the pituitary gland and pattern of release of the gonadotropin hormones, FSH and $\mathrm{LH}$, and the neural circuitries of courting, mating, and parenting. The discovery that both reproductive physiology and sexual behavior are differentiated as a result of prenatal production of androgens by the fetal testis in males, were made independently but more or less simultaneously in the late 1950s and early 1960s (see for review [4]). Since that time the empirical focus has been on determining the mechanisms of steroid action in sexual differentiation, beginning with determining where in the brain and what cell types express receptors for androgens and estrogens.

\section{STEROIDS PROGRAM MULTIPLE CELL TYPES}

Steroid hormones transduce their signals via receptors of the nuclear transcription factor family. Testosterone binds directly to the androgen receptor (AR), but is also a precursor hormone for both the potent androgen, dihydrotestosterone, and the estrogens estradiol, estriol, and estrone, all of which bind with varying affinities to the estrogen receptor (ER) isoforms, ER-alpha and ERbeta. Early studies of hormone action on the brain focused intently on the cellular distribution of these receptors with emphasis on brain region, cell type and the level of expression, which was predicted to be different in males and females. The thinking was that only a subset of cells, presumably neurons, expressed AR and $E R$, and these would be subject to hormonal programming and hence masculinization. Put differently, a small number of neurons would be sexually differentiated within a sea of cellular neutrality. Moreover, sexual differentiation would be restricted to those brain regions expressing high levels of $A R$ and ER. All of these

${ }^{1}$ Department of Pharmacology and Program in Neuroscience, University of Maryland School of Medicine, 655W. Baltimore St., Baltimore, MD 21201, USA

Correspondence: Margaret M. McCarthy (mmccarth@umaryland.edu)

Received: 5 April 2018 Revised: 1 June 2018 Accepted: 7 June 2018

Published online: 29 June 2018 
assumptions have been largely nullified. Neuroanatomical sex differences do not map in distribution or magnitude onto the distribution of steroid receptors. There are few and modest sex differences in the expression of $A R$ and ER, and they are functionally insignificant, since females can be fully masculinized by exogenous steroid. The levels of steroid in the developing brain also vary substantially by region, are often not sexually dimorphic and bear little to no relation to whether the region exhibits sex differences $[5,6]$. And, perhaps most importantly, sex differences are not limited to a few discrete cells. Instead, sex differences can be found in the morphology of entire populations of cells within a particular region, and can include neurons, astrocytes, and nonneuronal microglia [4].

Astrocytes are key partners in both the establishment of synapses during development, regulating their density and location, but also in adult plasticity. The rate at which astrocytes mature varies dramatically by brain region, at least as indicated by the expression of glia fibrillary acid protein (GFAP), a major structural protein that provides the complex ramified morphology characteristic of these cells. One of the more salient features of astrocytes is their localism. They occupy a very small space and that space usually ends where the territory of the next astrocyte begins. In this way astrocytes tile throughout the brain and respond only to what is happening to them and their nearest neighbors, be they neurons or other astrocytes. They are not just passive partners, however, as they vary in density and morphology by brain region or even subnucleus. They also vary between males and females in some regions and appear to confer regional specificity in neuronal morphology that also differs between males and females.

The arcuate nucleus resides in the ventral diencephalon and consists of neurons that receive feedback from neuronal populations responsive to internal physiology relevant to reproduction, growth, and metabolism. These neurons in turn regulate release of trophic hormones from the anterior pituitary and feedback to behavioral and physiological circuits within the brain. As noted above, control of gonadotropin release is markedly different in males and females, but so is growth hormone secretion and regulation of metabolism, making the arcuate an ideal brain region for investigation of neuroanatomical sex differences. Astrocytes in the arcuate mature early compared to other brain regions, with robust expression of GFAP. In neonatal males the astrocytes of the arcuate are larger and more stellate, with many and long processes, whereas in females they are simple and often bipolar in shape, with shorter and fewer processes [7]. When considering how this dimorphism in shape is established the first and most obvious question was, are they responsive to steroids? Treatment of neonatal females with estradiol or the precursor, testosterone, masculinizes the morphology of astrocytes to the point of being indistinguishable from that of males. This observation leads to the next question, are astrocytes directly responsive to steroids? For this to occur they require steroid receptors. While lack of evidence can never provide irrefutable evidence of lack, every attempt to identify steroid receptors in astrocytes of the arcuate nucleus has been negative [8]. This is not true for other brain regions, further attesting to the local identity of these cells. Regardless, since the morphology of astrocytes changes in response to steroid exposure, and the cells themselves do not seem to have steroid receptors, they must be responding to a signal from another cell that does respond directly to steroids, and in this case it is the neighboring neurons. Neurons of the arcuate nucleus express ER at high levels, and they are also largely GABAergic. Astrocytes express GABA-A receptors and due to a high intracellular chloride concentration, respond to GABA as an excitatory signal, resulting in increased intracellular calcium which induces process extension and branching. During the sensitive period for sexual differentiation, elevated estradiol in the male brain induces GABA synthesis and release from arcuate neurons which acts on neighboring astrocytes to induce a stellate morphology [9]. This change in astrocyte shape in turn correlates with a reduction in dendritic spine synapses on arcuate neurons, possibly via a physical suppression of synapse formation in a morphological feedback loop. The highly stellate morphology of male astrocytes is consistent with an increased "reactivity", meaning they are more excitable and potentially releasing more inflammatory mediators such as cytokines, although this has yet to be determined.

The preoptic area (POA), is also characterized by early maturation of astrocytes and here too they differ in morphology between males and females and drive a sex difference in dendritic morphology, only in the opposite direction [10]. The POA is often considered a subdivision of the hypothalamus due to its role in reproductive physiology and behavior but it is actually of telencephalic origin and an important embryonic source of GABAergic interneurons to the amygdala, hippocampus, and cortex [11]. The POA is arguably one of the most sexually differentiated brain regions, and it is certainly the most intensely studied as it is essential for both male copulatory behavior and female maternal behavior $[12,13]$. The sexually dimorphic nucleus (SDN) of the POA, a small Nissl dense subnucleus [14], is implicated in sexual partner preference [15], and has a homolog in the human hypothalamus [16].

As with the arcuate nucleus, the astrocytes of the male POA are more highly branched and stellate in morphology than those of the female [10], and similarly they do not appear to be directly responsive to steroids as they lack the requisite receptors. But there the similarity ends. Instead of GABA, the increased stellate morphology of astrocytes in males is driven by glutamate, presumably released from neighboring neurons that express ERalpha and ER-beta. Further contrasting the POA from the arcuate, astrocytes here work to build synaptic connections rather than repress them, and this is achieved via the surprising signal transduction molecule, prostaglandin E2 (PGE2) [17].

PGE2 is best known as an inflammatory molecule that promotes fever and is associated with the aches and pains of illness. It is a membrane-derived lipid from an arachidonic acid precursor via a multi-step enzymatic conversion that begins with the cyclooxygenase enzymes, COX-1 and COX-2. PGE2 is one of a class of prostanoids and is made ubiquitously throughout the brain and body both at basal levels and induced in response to insult or immune challenge. The primary action of non-steroidal antiinflammatory drugs (NSAIDs), such as aspirin, is inhibition of COX1 and COX-2, with the relative ratio of inhibition depending on the particular drug. The COX-1 isozyme is constitutively expressed while COX-2 is considered the inducible form, but this generalization does not appear to apply to the brain where both forms can be constitutive and both forms can be induced [18].

During the critical period for sexual differentiation of the brain, the mRNA and protein for both COX-1 and COX-2 are higher in the POA of males compared to females and this is paralleled by higher PGE2 content. If females are treated with a masculinizing dose of estradiol during the $48 \mathrm{~h}$ following birth, the COX enzymes and PGE2 levels increase to that of males by the 2nd day of life [19]. The estradiol-induced increase in COX enzymes, as well as the basal sex difference in PGE2 does not extend broadly to other brain regions as it is not observed in the hippocampus or amygdala. Local attributes of astrocytes may be an underlying aspect of the regional specificity but that remains to be determined. What has been determined, however, is the connection between PGE2 and dendritic morphology.

\section{NON-NEURONAL CELLS ARE SEXUALLY DIFFERENTIATED}

Astrocytes are not neurons but they share a common lineage and are epidermal in origin. The same is true for oligodendrocytes. The only true non-neuronal cells of the brain are those associated with 
40

the vasculature and the immune system. Microglia, which are unfortunately named because while they are small, they are large in number. Nor are they glia. Instead, microglia are the brains innate immune system. They enter the brain exceedingly early in development, day 9.5 in rodents and at the end of the first trimester in humans [20], which is prior to the majority of major developmental events, such as neurogenesis, differentiation, synaptogenesis, and myelination. This early arrival in the brain poises microglia to modulate many functions associated with maturation [21]. Similar to astrocytes, they tile throughout the brain and respond to local cues. Also similar to astrocytes, they exhibit sex differences in morphology and function in sexually differentiated brain regions.

The morphology of microglia is highly mutable and is a window into the physiological state of any given cell. While the shapes of microglia vary along a continuum, they can nonetheless be divided into categories for classification purposes. None of the divisions between categories are absolute, instead they provide a tool for quantifying how the activation status of microglia might vary across age, within brain regions and between the sexes. Those studies which have examined the progression of microglia morphology across ages find that early in life they are more likely to have an ameboid-like shape [22, 23]. This does not mean they are moving, but rather they have few if any processes and appear more blob-like than the opposite extreme, which is a fully ramified shape characterized by a small cell body and long thin highly branched processes. In the mature brain, the more blob-like in shape a particular microglia is the more "activated" it is considered, meaning it produces higher levels of inflammatory mediators and immune cell markers, such as prostaglandins and inflammatory cytokines. By contrast, cells with a morphology that is highly ramified are considered un-activated, but this does not mean they are not doing anything. Prior to live cell imaging, that is what was believed, and they were referred to as "quiescent" microglia, thought to be sitting in quiet anticipation of an accident or insult at which time they would leap into action. Once cells could be imaged in living tissue, however, it became quickly apparent that microglia have highly motile processes and that those in a ramified morphology are actually checking on their nearest neighbors and even physically touching neurons [24] and individual synapses [25]. Microglia in this morphology are now referred to as "surveying". More recently a 1-5 scale of microglial activational state has been established based on a combination of morphology and expression of lysosomal enzymes [26]. An important question is whether microglia that appear activated in the developing brain are really just immature, as indeed the average morphology of microglia becomes more ramified as development progresses $[23,27]$. For at least one brain region, the POA, it appears that the more ameboid-like microglia in the male are indicative of a higher activational state [28].

As immune cells, microglia both respond to and make prostaglandins and can be contributors to both deleterious runaway inflammation after an injury, as well as forces for good by clearing out cellular debris and releasing anti-inflammatory cytokines [29-31]. In the POA the cellular source of higher PGE2 in males was unknown but microglia were certainly one viable candidate. Morphological characterization of POA microglia during the sensitive period for sexual differentiation revealed male cells to be both more common (i.e. higher density) and on average to exhibit a more ameboid-like or activated state. Treating females with a masculinizing dose of estradiol increased both the number and activation state of resident microglia to that of males, and more importantly correspondingly increased PGE2 production. If the females were simultaneously treated with the microglial inhibitor, minocycline, none of these responses occurred, included the increase in PGE2, thereby confirming that these immune cells are a critical source of prostaglandin for developmental masculinization of the POA [28]. Moreover, if microglia were chemically depleted in the POA during the critical period of sexual differentiation, as adult males were completely void of any mating behavior, showing no interest in either male or female conspecifics [32].

Dendrites of POA neurons are relatively simple, with few primary branches and even fewer secondary ones. They are, however, studded with dendritic spines, presumptive sites of excitatory synapses. In males, the density of dendritic spines is roughly twice that of females, meaning per unit of dendrite there is double the amount of excitatory input [17]. The synaptic input to POA neurons is at the end of a chain of connections beginning with the main and accessory olfactory bulbs passing through the amygdala and bed nucleus of the stria terminalis (BNST). Through this synaptic circuit olfactory and pheromonal signals are integrated to impact on the excitability of POA neurons, which in turn contribute to the behavioral outputs of male mating behavior and female maternal behavior. These are adult behaviors but the double density of synapses in males is established during the critical period for sexual differentiation at and around birth, when PGE2 levels are markedly higher in male POA compared to female POA. So how do we connect a prostaglandin to synapse formation?

\section{INFLAMMATORY MOLECULES INITIATE KEY SIGNALING CASCADES}

The principle receptors for PGE2 are EP1-4 and are GPCRs that differentially couple with adenyl cyclase depending on isoforms and dimerization [33]. Both EP2 and EP4 are stimulatory to production of CAMP and subsequent activation of protein kinase $A$ (PKA). The synapses at spines are glutamatergic and usually a mix of NMDA and AMPA. The localization of AMPA receptors to the post-synaptic density is dependent upon phosphorylation of particular subunits, thereby allowing the receptor to cycle in and out of the membrane and the synapse as a function of neuronal excitation or other parameters. PGE2 stimulates CAMP production and PKA activation in POA neurons, leading to phosphorylation of the critical AMPA receptor subunit that traffics the receptor to the membrane and presumably the synapse [34]. When this occurs during the critical period it leads to the formation and stabilization of dendritic spines, the precise details of which remain unknown but the enduringness of is not in dispute. The formation and maintenance of this pattern of spine density is essential for the ability of adult males to respond to olfactory cues from females and pursue mating [35].

Sex differences in microglial number and morphology have been reported for other brain regions as well, including the amygdala and hippocampus [36-38]. In these instances, a direct connection to sex differences in adult behavior has yet to be established. But a role for microglia in transducing the enduring impact of developmental opioid exposure has been [39], and reflects the vital role the innate immune system plays in integrating early experience into later outcome.

\section{EPIGENETIC PROGRAMMING REPRESSES INFLAMMATORY SIGNALING}

Most, if not all, early life programming is now understood to be secondary to epigenetic modifications of the genome, which commonly occurs via methylation of cytosine nucleotides, as well as histone tails that also accept and release acetyl groups and other modifiers. In order to address whether epigenetics underpin the enduring consequences of hormonally mediated sexual differentiation, the DNA methylome of the POA was compared in newborn rat pups. Females were found to have higher levels of methylation of CpG throughout the genome [40], suggesting there is a set of genes that are strongly epigenetically suppressed, but that this suppression is less so in males. In order to gain 

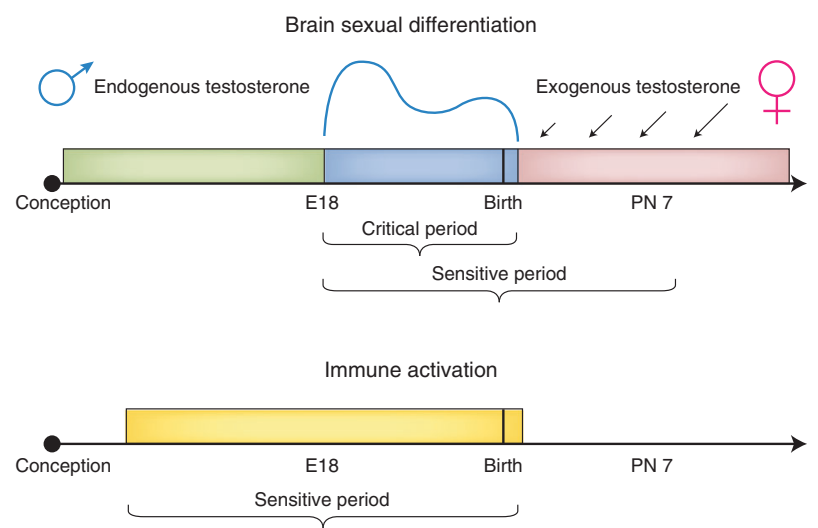

Fig. 1 Sensitive and critical periods in brain development. The developing brain is characterized by epochs during which essential stimuli direct cell survival and synaptic patterns, thereby programming the brain during a critical period. Sensitive periods may overlap with critical periods but also include times during which aberrant or exogenous stimuli can alter the normal developmental trajectory. Sexual differentiation of the brain includes a critical period during which endogenous testosterone from the fetal testis masculinizes the brain. Females remain sensitive to the masculinizing effects of exogenous testosterone into the first week of life, albeit it requiring increasingly larger doses (indicated by larger arrows). The brain is also sensitive to immune activation, either via sickness in the mother or directly to the newborn, during a restricted period that is still being understood. Importantly, the critical period for masculinization of the brain and the sensitive period to inflammation overlap and may contribute to sex differences in risk of developmental neuropsychiatric disorders insight into what those genes are, newborn females were treated with an inhibitor of DNMT activity, thereby reducing DNA methylation. Examination of the transcriptome in females with the normal methylation pattern versus those treated with the DNMT inhibitor revealed that a cohort of genes involved in immune regulation was normally suppressed by epigenetic modifications [40]. This is consistent with the observed up regulation of immune cells and inflammatory mediators found in the developing male POA [41]. An important question is how it comes to be that the DNA in cells of the POA are less methylated in males (Fig. 1). Treatment of females with a masculinizing dose of estradiol (the masculinizing hormone in rodents) reduces DNMT activity and DNA methylation in parallel but whether this unmasks the same cohort of immune responsive genes is not yet known. Steroids are generally considered anti-inflammatory, making them an unlikely candidate for driving increases in immune-related gene expression. As discussed below, another possibility is that steroids are actually a counter protective agent and that the higher level of immune activation in the male brain is a byproduct of the maternal immune system being more active against male fetuses than female fetuses.

\section{MICROGLIAL PRIMING AS A SOURCE OF IMMUNE MEMORY}

Priming refers to when an innate immune cell is exposed to a pathogen or toxin, and upon re-exposure shows an enhanced response, such as higher cytokine production than witnessed following the first exposure [42]. The innate immune system is not generally considered to be the source of immunological memory, that is a job left to the acquired immune system, as the name suggests. But the phenomenon of priming has prompted

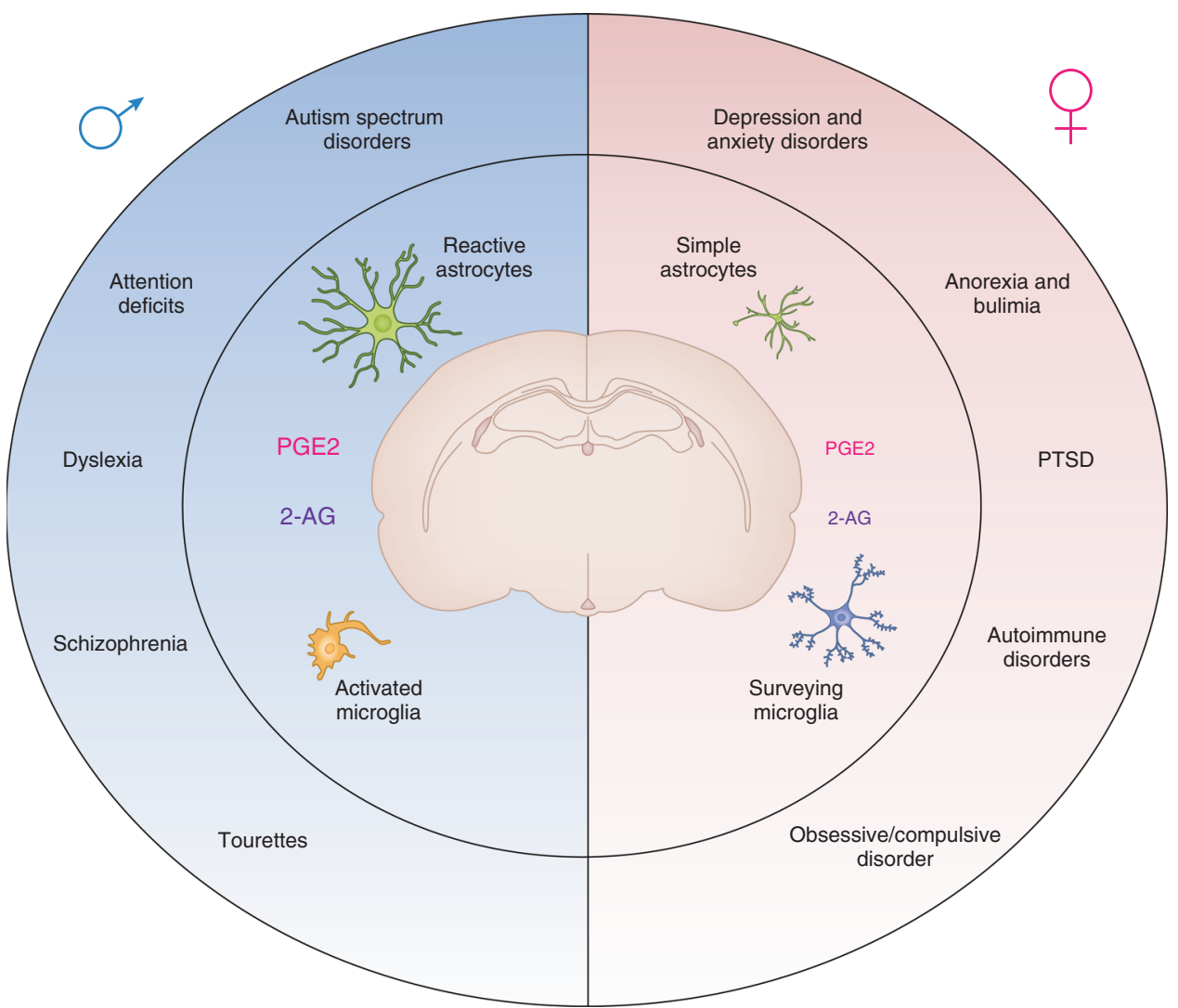

Fig. 2 Higher inflammation in developing male brains as an inherent risk factor. Cellular mechanisms mediating masculinization of the brain frequently involve elevated levels of inflammatory signaling molecules and immune cells. Early life exposure to inflammation is a documented risk factor for developmental neuropsychiatric disorders. Being male is also a documented risk factor, suggesting there may be a convergence in the normal process of masculinization and environmental events increasing risk. PGE2 prostaglandin E2, 2-AG endocannabinoid 
immunologist to rethink this dichotomy. In the periphery, the priming response is mediated by permissive epigenetic modifications of latent enhancers in the promoters of pro-inflammatory genes, resulting in greater expression upon second exposure. Microglia are innate immune cells and are known to be activated following exposure to pathogens or toxins, leading to the suggestion that this is also a case of priming [43]. The priming response can last for weeks to months, meaning the majority of the lifetime of a mouse. This raises the interesting question of whether a similar phenomenon might happen in the developing brain and whether microglia in males experience a stronger priming effect resulting in a more robust production of prostaglandins and other inflammatory signaling molecules into adulthood. This priming may be related to the epigenetic modifications noted above. One of our future challenges will be to knit these disparate observations into a coherent fabric.

\section{SEX DIFFERENCES IN NEUROINFLAMMATION}

With increasing confidence, it can be asserted that inflammation during early brain development contributes to the life time risk for a neuropsychiatric disorder. Evidence is strongest for schizophrenia and growing for autism spectrum disorders (ASDs) [44]. Equally strong clinical and epidemiological data reveal the higher diagnosis rates in males for both autism and schizophrenia [45, 46], albeit with multiple caveats regarding presentation, onset, severity, etc. The maternal immune activation (MIA) model in rodents and non-human primates has proven a powerful tool for exploring the origins of developmental neuropsychiatric disorders [47], but has not consistently considered the potential influence of sex. Thus, it is time to ask if these two biological variables, maleness and neuroinflammation, converge to increase risk (Fig. 2). One can envision two means by which this could occur. First is that under healthy conditions males and females are identical, but that under inflammatory conditions something inherent in maleness results in greater vulnerability. Alternatively, males could inherently have higher inflammatory tone, which puts them at greater risk when an additional inflammatory or other insult occurs. The data generated in animal models mostly suggest the latter, that developing males have an inherently higher inflammatory tone. But much more importantly, so does data in humans.

ASDs are among the most strongly gender biased of neuropsychiatric disorders [46] and perinatal inflammation is one of the strongest associated risk factors [44, 48, 49]. However, few have combined these two variables. A re-analyses of transcriptome data from publically available data bases by Werling and colleagues observed that the transcriptomic profile of fetal male cortex was more indicative of inflammation, based on activated astrocytes and microglia, compared to fetal females [50]. This seemed surprising but when placed in the context of the existing animal literature is highly consistent. Werling and colleagues went on to compare post-mortem adult male cortical transcriptomes and found that those derived from individuals with an autism diagnosis had even higher indices of inflammation compared to those without. Whether the higher inflammatory indices in adult male autistics is a cause or a consequence of living with autism is unknown but together with the data from fetal brains these findings are consistent with the notion of an inherently higher risk in males that originates with inflammation.

Inflammation in the brain has become of broad interest for a wide range of conditions including traumatic brain injuries, such as concussion, neurodegenerative disorders such as Alzheimer's and even major depressive disorder [51]. Depression is almost twice as frequently diagnosed in woman as men $[52,53]$, as are a number of other post-pubertal onset disorders including anorexia nervosa, general anxiety disorder, obsessive compulsive disorder and the autoimmune diseases multiple sclerosis and lupus [54].
While no sweeping generalizations can be made regarding sex differences in the adult immune system, on average the immune response of adult females is more robust than that of males [55], suggesting the reversal of fortunes in gender bias from development to adulthood may have its origins at least in part in the immune system.

\section{PERIPHERAL SOURCES OF INFLAMMATION MAY IMPACT THE DEVELOPING BRAIN}

The association of maternal illness and immune activation with later risk for developmental neuropsychiatric disorders highlights the impact of extremely early events occurring long before the brain has fully formed. This puzzling fact suggests that tissues outside the nervous system, and perhaps even the body, may be key to participants in generating risk. This suggestion is borne out in recent work implicating the placenta as a transducer of stress and other signals to the developing fetus [56]. The placenta was more closely tied to schizophrenia by deriving polygenic risk scores (PRS) from large Genome Wide Association Studies (GWAS). Mining medical records and patient interviews, the presence of complications during pregnancy or birth (pre-eclampsia, prematurity, very low birth weight, etc.) the liability of schizophrenia explained by the highest PRS scores was increased five-fold [57]. The genes found in the highest PRS were enriched in the placenta and associated with oxidative stress and metabolism, and not neuronal development per se. When placenta from male versus female fetuses that experienced pregnancy complications were compared, the schizophrenia-risk genes were higher in males, suggesting a more robust dysregulation in developing boys.

The placenta is a temporary organ and how or if it influences the brain as development progresses postnatally is unknown. In general the trafficking of immune cells into and out of the brain from the periphery is still poorly understood [58], and we are only just beginning to gain a hold on the diversity of neural immune cells [59]. For instance, a class of immune cells, the peripheral leukocyte B-1a cell, has been implicated in promoting myelination by increasing the proliferation of oligodendrocytes in the developing corpus callosum [60]. The B-cells are attracted to the brain from the blood by secretion of chemoattractant cytokine from the choroid plexus and meninges of the lateral ventricles. Secretion of the immunoglobulin IgM by the B-cells stimulates neuronal stem cells to differentiate into oligodendrocytes. Depletion of B-1a cells impairs appropriate myelination, a process that has also been associated with hypoxic/ischemic brain damage following birth complications and in developmental neuropsychiatric disorders, such as ASD and schizophrenia, all of which are more frequent or more severe in males [46, 61, 62]. Much about this particular process remains to be learned, nonetheless, it demonstrates the potential for sensitive windows of development during which the immune and nervous system are engaged in a critical conversation directing how the brain is constructed.

Mast cells are also a peripheral immune cell as they are found throughout the body, but can also migrate into the brain following a frank injury, but also in response to other as yet unknown stimuli [63]. The resident mast cells are few in number but capable of integrating peripheral signals and have a mutually communicative relationship with microglia [64]. Dysregulation of mast cells, both in the periphery and centrally have been suggested as causative factors in ASD [65], highlighting the potentially powerful impact of just one type of immune cell.

\section{MATERNAL ANTIBODIES IMPACT THE DEVELOPING BRAIN}

Additional insights into the increased risk for boys and young men comes from a completely different quarter, the maternal immune hypothesis of male homosexuality [66]. Blanchard and colleagues 
have long reported that the frequency of men with a sexual preference for men positively correlates with the number of older brothers, but is independent of the number of older sisters. Moreover, the birth weight of boys with older brothers is lower and lower still in those that eventually identify as homosexual, suggesting more vigorous MIA towards the developing male fetus [66]. Evidence that this is the case was found in analyses of serum antibodies against neuroligin 4Y-linked, a gene coding for a protein integral to synapse formation. Women overall had higher titers of antibodies against neuroglin4Y compared to men. Women who had given birth to sons had higher titers still but the highest levels were found in women who had gay sons [67]. Precisely how this alters the trajectory of brain development and impacts on sexual preference is unknown but it demonstrates the potential for additional maternal immune reactions that are more subtle and complex than those initiated by sickness. Similarly, the appearance of maternal antibodies in the serum of newborn infants has been associated with ASD and schizophrenia [68]. Particularly intriguing is the apparent propensity of the maternal immune system to react to proteins of the fetal brain [69], although whether this is an observational bias due to over emphasis of the nervous system, remains to be determined.

\section{FUTURE DIRECTIONS}

In the continuing quest to unearth the myriad factors that put an individual at risk for developing a neuropsychiatric disorder, the importance of two variables is being continuously reinforced; being male and exposure to inflammation during critical periods. There has been insufficient attention to the convergence of these two variables as we lack an understanding of how they relate to each other. To-date there is no clear epidemiological or clinical data indicating that early life inflammation selectively increases risk in males but this may be because the question has not been properly asked. Instead the emphasis has been on traditional variables of maleness, most notably fetal androgen levels [70], which has met with limited success [71]. Whether fetal steroids are orthogonal to fetal neuroinflammation or a contributing variable, either in promoting or dampening, is not known. In other words, are there multiple sources of vulnerability to males that include steroids from the fetal gonads, genes on the $X$ and $Y$ chromosome and possibly the maternal immune system? Or, is there a final common pathway onto which all the aspects of maleness converge to increase overall risk. Moreover, how does that risk manifest across a wide range of disparate disorders from autism to stuttering to attention deficits to schizophrenia. We have much work to do.

\section{ACKNOWLEDGEMENTS}

This work was supported by R01DA039062 and RO1MH52716 to MMM.

\section{ADDITIONAL INFORMATION}

Competing interests: The authors declare no competing interests.

Publisher's note: Springer Nature remains neutral with regard to jurisdictional claims in published maps and institutional affiliations.

\section{REFERENCES}

1. Patterson PH. Maternal infection: window on neuroimmune interactions in fetal brain development and mental illness. Curr Opin Neurobiol. 2002;12:115-8.

2. McCarthy MM, Wright CL. Convergence of sex differences and the neuroimmune system in autism spectrum disorder. Biol Psychiatry. 2017;81:402-10. https://doi. org/10.1016/j.biopsych.2016.10.004

3. Goodfellow PN, Lovell-Badge R. SRY and sex determination in mammals. Annu Rev Genet. 1993;27:71-92. https://doi.org/10.1146/annurev.ge.27.120193.000443
4. McCarthy MM, De Vries GJ, Forger NG. Sexual differentiation of the brain: a fresh look at mode, mechanisms and meaning. In: D. a. J. Pfaff $M$, (Ed.). Hormones, brain and behavior. vol. 3. San Diego: Elsevier; 2017. p. 3-32.

5. Konkle AT, McCarthy MM. Developmental time course of estradiol, testosterone, and dihydrotestosterone levels in discrete regions of male and female rat brain. Endocrinology. 2011;152:223-35. doi: en.2010-0607 [pii] 10.1210/en. 2010-0607

6. Amateau SK, Alt JJ, Stamps CL, McCarthy MM. Brain estradiol content in newborn rats: sex differences, regional heterogeneity, and possible de novo synthesis by the female telencephalon. Endocrinology. 2004;145:2906-17.

7. Mong JA, McCarthy MM. Ontogeny of sexually dimorphic astrocytes in the neonatal rat arcuate. Dev Brain Res. 2002;139:151-8.

8. McCarthy MM, Amateau SK, Mong JA. Steroid modulation of astrocytes in the neonatal brain: Implications for adult reproductive function. Biol Reprod. 2002;67:691-8.

9. Mong JA, Nunez JL, McCarthy MM. GABA mediates steroid-induced astrocyte differentiation in the neonatal rat hypothalamus. J Neuroendo. 2002;14:1-16.

10. Amateau SK, McCarthy MM. Sexual differentiation of astrocyte morphology in the developing rat preoptic area. J Neuroendo. 2002;14:904-10.

11. Gelman DM, Martini FJ, Nobrega-Pereira S, Pierani A, Kessaris N, Marin O. The embryonic preoptic area is a novel source of cortical GABAergic interneurons. J Neurosci. 2009;29:9380-9. https://doi.org/10.1523/JNEUROSCI.0604-09.2009

12. Hull EM, Dominguez JM. Sexual behavior in male rodents. Horm Behav. 2007;52:45-55. https://doi.org/10.1016/j.yhbeh.2007.03.030

13. Lonstein JS, Greco B, De Vries GJ, Stern JM, Blaustein JD. Maternal behavior stimulates c-fos activity within estrogen receptor alpha-containing neurons in lactating rats. Neuroendocrinology. 2000;72:91-101.

14. Gorski RA, Gordon JH, Shryne JE, Southam AM. Evidence for a morphological sex difference within the medial preoptic area of the rat brain. Brain Res. 1978;148:333-46.

15. Roselli CE, Larkin K, Resko JA, Stellflug JN, Stormshak F. The volume of a sexually dimorphic nucleus in the ovine medial preoptic area/anterior hypothalamus varies with sexual partner preference. Endocrinology. 2004;145:478-83.

16. LeVay S. A difference in hypothalamic structure between heterosexual and homosexual men. Science. 1991;9:497-506.

17. Amateau SK, McCarth MM. A novel mechanism of dendritic spine plasticity involving estradiol induction of prostglandin-E2. J Neurosci. 2002;22:8586-96.

18. Kaufmann W, Andreasson K, Isakson P, Worley P. Cyclooxygenases and the central nervous system. Prostaglandins. 1997;54:601-24.

19. Amateau SK, McCarthy MM. Induction of PGE(2) by estradiol mediates developmental masculinization of sex behavior. Nat Neurosci. 2004;7:643-50.

20. Ginhoux F, Lim S, Hoeffel G, Low D, Huber T. Origin and differentiation of microglia. Front Cell Neurosci. 2013;7:45 https://doi.org/10.3389/fncel.2013.00045

21. Nayak D, Roth TL, McGavern DB. Microglia development and function. Annu Rev Immunol. 2014;32:367-402. https://doi.org/10.1146/annurev-immunol-032713120240

22. Bilbo SD, Schwarz JM. The immune system and developmental programming of brain and behavior. Front Neuroendocrinol. 2012;33:267-86. https://doi.org/ 10.1016/j.yfrne.2012.08.006

23. Perez-Pouchoulen M, VanRyzin JW, McCarthy MM. Morphological and phagocytic profile of microglia in the developing rat cerebellum. eNeuro, 2015;2. https://doi. org/10.1523/ENEURO.0036-15.2015

24. Nimmerjahn A, Kirchhoff $F$, Helmchen F. Resting microglial cells are highly dynamic surveillants of brain parenchyma in vivo. Science. 2005;308:1314-8. https://doi.org/10.1126/science.1110647

25. Weinhard L, di Bartolomei G, Bolasco G, Machado P, Schieber NL, Neniskyte U, Gross CT. Microglia remodel synapses by presynaptic trogocytosis and spine head filopodia induction. Nat Commun. 2018;9:1228 https://doi.org/10.1038/ s41467-018-03566-5

26. Hong S, Dissing-Olesen L, Stevens B. New insights on the role of microglia in synaptic pruning in health and disease. Curr Opin Neurobiol. 2016;36:128-34. https://doi.org/10.1016/j.conb.2015.12.004

27. Hanamsagar R, Alter MD, Block CS, Sullivan H, Bolton JL, Bilbo SD. Generation of a microglial developmental index in mice and in humans reveals a sex difference in maturation and immune reactivity. Glia. 2017. https://doi.org/10.1002/glia.23176

28. Lenz KM, Nugent BM, Haliyur R, McCarthy MM. Microglia are essential to masculinization of brain and behavior. J Neurosci. 2013;33:2761-72.

29. Akundi RS, Candelario-Jalil E, Hess S, Hull M, Lieb K, Gebicke-Haerter PJ, Fiebich BL. Signal transduction pathways regulating cyclooxygenase- 2 in lipopolysaccharideactivated primary rat microglia. Glia. 2005;51:199-208. https://doi.org/10.1002/ glia.20198

30. Quan Y, Jiang J, Dingledine R. EP2 receptor signaling pathways regulate classical activation of microglia. J Biol Chem. 2013;288:9293-302. https://doi.org/10.1074/ jbc.M113.455816 
31. Taylor RA, Sansing LH. Microglial responses after ischemic stroke and intracerebral hemorrhage. Clin Dev Immunol. 2013;2013:746068 https://doi.org/10.1155/ 2013/746068

32. VanRyzin, JW, Yu, SJ, Perez-Pouchoulen, M, McCarthy, MM. Temporary depletion of microglia during the early postnatal period induces lasting sex-dependent and sex-independent effects on behavior in rats. eNeuro. 2016;3. https://doi.org/ 10.1523/ENEURO.0297-16.2016

33. Regan JW. EP2 and EP4 prostanoid receptor signaling. Life Sci. 2003;74:143-53.

34. Lenz KM, Wright CL, Martin RC, McCarthy MM. Prostaglandin E regulates AMPA receptor phosphorylation and promotes membrane insertion in preoptic area neurons and glia during sexual differentiation. PLoS ONE. 2011;6:e18500 https:// doi.org/10.1371/journal.pone.0018500

35. Wright CL, McCarthy MM. Prostaglandin E2-induced masculinization of brain and behavior requires protein kinase A, AMPA/kainate, and metabotropic glutamate receptor signaling. J Neurosci. 2009;29:13274-82.

36. Nelson LH, Warden S, Lenz KM. Sex differences in microglial phagocytosis in the neonatal hippocampus. Brain Behav Immun. 2017;64:11-22. https://doi.org/ 10.1016/j.bbi.2017.03.010

37. Schwarz J, Sholar PW, Bilbo SD. Sex differences in microglial colonization of the developing rat brain. J Neurochem. 2012;120:948-63.

38. Weinhard L, Neniskyte U, Vadisiute A, di Bartolomei G, Aygun N, Riviere L, et al. C. Sexual dimorphism of microglia and synapses during mouse postnatal development. Dev Neurobiol. 2017. https://doi.org/10.1002/dneu.22568

39. Schwarz JM, Bilbo SD. Adolescent morphine exposure affects long-term microglial function and later-life relapse liability in a model of addiction. J Neurosci. 2013;33:961-71. https://doi.org/10.1523/JNEUROSCI.2516-12.2013

40. Nugent BM, Wright CL, Shetty AC, Hodes GE, Lenz KM, Mahurkar A, McCarthy $M M$. Brain feminization requires active repression of masculinization via DNA methylation. Nat Neurosci. 2015;18:690-7. https://doi.org/10.1038/nn.3988

41. McCarthy MM, Nugent BM, Lenz KM. Neuroimmunology and neuroepigenetics in the establishment of sex differences in the brain. Nat Rev Neurosci. 2017;18:471-84. https://doi.org/10.1038/nrn.2017.61

42. Netea MG, Joosten LA, Latz E, Mills KH, Natoli G, Stunnenberg HG, Xavier RJ. Trained immunity: A program of innate immune memory in health and disease. Science. 2016;352:aaf1098 https://doi.org/10.1126/science.aaf1098

43. Haley MJ, Brough D, Quintin J, Allan SM. Microglial priming as trained immunity in the brain. Neuroscience. 2017. https://doi.org/10.1016/j.neuroscience.2017.12.039

44. Estes ML, McAllister AK. Maternal immune activation: implications for neuropsychiatric disorders. Science. 2016;353:772-7. https://doi.org/10.1126/science. aag3194

45. Abel KM, Drake R, Goldstein JM. Sex differences in schizophrenia. Int Rev Psychiatry. 2010;22:417-28. https://doi.org/10.3109/09540261.2010.515205

46. Werling DM. The role of sex-differential biology in risk for autism spectrum disorder. Biol Sex Differ. 2016;7:58 https://doi.org/10.1186/s13293-016-0112-8

47. Patterson PH. Immune involvement in schizophrenia and autism: etiology, pathology and animal models. Behav Brain Res. 2009;204:313-21. https://doi.org/ 10.1016/j.bbr.2008.12.016

48. Nardone $S$, Elliott $E$. The interaction between the immune system and epigenetics in the etiology of autism spectrum disorders. Front Neurosci. 2016;10:329 https:// doi.org/10.3389/fnins.2016.00329

49. Spencer SJ, Meyer U. Perinatal programming by inflammation. Brain Behav Immun. 2017;63:1-7. https://doi.org/10.1016/j.bbi.2017.02.007

50. Werling DM, Parikshak NN, Geschwind DH. Gene expression in human brain implicates sexually dimorphic pathways in autism spectrum disorders. Nat Commun. 2016;7:10717 https://doi.org/10.1038/ncomms10717

51. Dantzer R, O'Connor JC, Freund GG, Johnson RW, Kelley KW. From inflammation to sickness and depression: when the immune system subjugates the brain. Nat Rev Neurosci. 2008;9:46-56. https://doi.org/10.1038/nrn2297
52. Kessler RC. Epidemiology of women and depression. J Affect Disord. 2003;74:5-13.

53. Altemus M. Sex differences in depression and anxiety disorders: potential biological determinants. Horm Behav. 2006;50:534-8. https://doi.org/10.1016/j. yhbeh.2006.06.031

54. McCarthy MM. Multifaceted origins of sex differences in the brain. Philos Trans $R$ Soc Lond B 2016;371. https://doi.org/10.1098/rstb.2015.0106

55. vom Steeg LG, Klein SL. SeXX matters in infectious disease pathogenesis. PLoS Pathog. 2016;12:e1005374 https://doi.org/10.1371/journal.ppat.1005374

56. Howerton $\mathrm{CL}$, Morgan CP, Fischer DB, Bale TL. O-GlcNAc transferase (OGT) as a placental biomarker of maternal stress and reprogramming of CNS gene transcription in development. Proc Natl Acad Sci USA. 2013;110:5169-74. https://doi. org/10.1073/pnas.1300065110

57. Ursini G, Punzi G, Chen Q, Marenco S, Robinson JF, Porcelli A, Weinberger DR. Convergence of placenta biology and genetic risk for schizophrenia. Nat Med. 2018. https://doi.org/10.1038/s41591-018-0021-y

58. Kipnis J. Multifaceted interactions between adaptive immunity and the central nervous system. Science. 2016;353:766-71. https://doi.org/10.1126/science. aag2638

59. Mrdjen D, Pavlovic A, Hartmann FJ, Schreiner B, Utz SG, Leung BP, Becher B. Highdimensional single-cell mapping of central nervous system immune cells reveals distinct myeloid subsets in health, aging, and disease. Immunity. 2018;48:599 https://doi.org/10.1016/j.immuni.2018.02.014

60. Tanabe S, Yamashita T. B-1a lymphocytes promote oligodendrogenesis during brain development. Nat Neurosci. 2018;21:506-16. https://doi.org/10.1038/ s41593-018-0106-4

61. Hill CA, Fitch RH. Sex differences in mechanisms and outcome of neonatal hypoxia-ischemia in rodent models: implications for sex-specific neuroprotection in clinical neonatal practice. Neurol Res Int. 2012;2012:867531 https://doi.org/ 10.1155/2012/867531

62. Aleman A, Kahn RS, Selten JP. Sex differences in the risk of schizophrenia: evidence from meta-analysis. Arch Gen Psychiatry. 2003;60:565-71. https://doi.org/ 10.1001/archpsyc.60.6.565

63. Silverman AJ, Sutherland AK, Wilhelm M, Silver R. Mast cells migrate from blood to brain. J Neurosci. 2000;20:401-8.

64. Skaper SD, Giusti P, Facci L. Microglia and mast cells: two tracks on the road to neuroinflammation. FASEB J. 2012;26:3103-17. https://doi.org/10.1096/fj.11197194

65. Theoharides TC, Asadi S, Patel AB. Focal brain inflammation and autism. J Neuroinflamm. 2013;10:46 https://doi.org/10.1186/1742-2094-10-46

66. Blanchard R. Fraternal birth order and the maternal immune hypothesis of male homosexuality. Horm Behav. 2001;40:105-14. https://doi.org/10.1006/ hbeh.2001.1681

67. Bogaert AF, Skorska MN, Wang C, Gabrie J, MacNeil AJ, Hoffarth MR, Blanchard R. Male homosexuality and maternal immune responsivity to the $\mathrm{Y}$-linked protein NLGN4Y. Proc Natl Acad Sci USA. 2018;115:302-6. https://doi.org/10.1073/ pnas.1705895114

68. Kowal C, Athanassiou A, Chen H, Diamond B. Maternal antibodies and developing blood-brain barrier. Immunol Res. 2015;63:18-25. https://doi.org/10.1007/s12026015-8714-5

69. Braunschweig D, Van de Water J. Maternal autoantibodies in autism. Arch Neurol. 2012;69:693-9. https://doi.org/10.1001/archneurol.2011.2506

70. Auyeung B, Baron-Cohen S, Ashwin E, Knickmeyer R, Taylor K, Hackett G. Fetal testosterone and autistic traits. Br J Psychol. 2009;100:1-22. 298715 [pii] 10.1348/ 000712608 X311731

71. Baron-Cohen S, Auyeung B, Norgaard-Pedersen B, Hougaard DM, Abdallah MW, Melgaard L, Lombardo MV. Elevated fetal steroidogenic activity in autism. Mol Psychiatry. 2015;20:369-76. https://doi.org/10.1038/mp.2014.48 